\title{
Biomechanical Evaluation of the Mandibular First Molar After Simulated Occlusal Wear Using 3D Finite Element Analysis
}

\section{Ke Guo}

Shanghai Jiao Tong University School of Medicine

\section{Shoufu Sun}

Shanghai Jiao Tong University School of Medicine

\section{Yueqi Shi}

Shanghai Jiao Tong University School of Medicine

Ying Zhang

Shanghai Jiao Tong University School of Medicine

Wenjia Wei

Shanghai Jiao Tong University School of Medicine

Haoming Zhao ( $\sim$ ZHM4586@shtrhospital.com)

Shanghai Jiao Tong University School of Medicine

\section{Research Article}

Keywords: Mandibular first molar, abrasion, three-dimensional finite element, equivalent stress, instantaneous displacement

Posted Date: January 4th, 2022

DOI: https://doi.org/10.21203/rs.3.rs-1213849/v1

License: (9) This work is licensed under a Creative Commons Attribution 4.0 International License. Read Full License 


\section{Abstract}

PURPOSEXThis study aimed to investigate the biomechanical basis of vertical tooth fracture occurring in the mesial root of the mandibular first molar.

METHODS $\varangle$ We used 3D finite elements to analyze the stress distribution and transient displacement of the mandibular first molar after occlusal surface wear and tooth tilt. Based on four degrees of wear within each of the surface wear and tooth tilt groups, eight models were established in addition to the control model. A simulated bite force of $200 \mathrm{~N}$ was loaded on the occlusal surface, and nonlinear finite element analysis was used to explore the biomechanical basis of vertical root fracture.

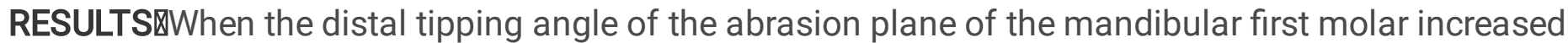
from $5^{\circ}$ to $15^{\circ}$, the angle between the instantaneous displacement contours and the long axis of the tooth decreased. Meanwhile, the mesial root was found to suffer the highest stress concentration, and the possibility of longitudinal root fracture was increased.

CONCLUSION: By evaluating the biomechanical effect of tooth wear and occlusal loading, we are able to identify some clinical interventions that may prevent vertical tooth fracture.

\section{Introduction}

Vertical root fractures (VRFs) refer to fractures of the tooth root along the longitudinal axis, through the medullary cavity and the pericementum space [1]. These fractures may originate anywhere on or within the root, may extend coronally toward the cervical margin in the facial or lingual aspects, and are extremely challenging to diagnose in dental practice $[2,3]$. In fact, the mandibular first molar, which is possibly exposed to the maximum biting force, commonly suffers from VRFs because of severe tooth abrasion [4].

In recent years, VRFs, especially in the essential teeth, have received increased attention. Detection of VRFs in their initial stages is crucial, because it can help prevent the propagation of injury to the adjacent supporting structures; once the injury is propagated it is impossible to save the tooth [5]. The incidence of VRFs after root canal treatment varies between $8.8 \%$ and $31 \%[6,7]$. Early detection of VRFs needs very careful consideration. Most of the patients visit the dentist when they have obvious symptoms, most of whom must undergo tooth extraction. As a result, biomechanical research regarding the etiopathophysiology of VRFs, especially with regard to tooth abrasion, is needed.

Severe tooth abrasion, or the loss of dental hard tissues including enamel and dentin, is the most common non-carious dental abnormality, and it can seriously affect oral health [8]. It is commonly caused by direct contact between opposing teeth or between teeth and foreign objects [9], and can be divided into the following subtypes: mechanical wear (attrition and abrasion) and chemical wear (erosion) [10]. Recent research has called for quantitative study of tooth abrasion to improve upon descriptive methods and indices for the recognition of attrition $[11,12]$. 
Tooth wear commonly causes the tilting of a tooth's biting surface towards the distal side. Due to this tilt, the occlusal surface becomes the distal surface after a tooth is worn-out. The studies focused on the stress distribution of the mesial root have mostly been related to normal abrasion or horizontal abrasion and rarely related to tilt abrasion. Thus, we aimed to establish models with different tilt angles towards the distal side to analyze the impact of inclined wear on the mesial root. By studying the tooth biomechanically, we aimed to characterize the risk factors for mesial root vertical fractures.

In this study, three-dimensional finite element analysis was used to simulate the horizontal and tilt abrasion of the permanent mandibular first molar to investigate the distribution of stress and transient displacement brought about by maxillomandibular loading and to provide a biomechanical basis for reducing the possibility of VRFs.

\section{Materails And Methods}

\section{Subjects}

A healthy male volunteer (18 years old), whose mandibular first molar showed well-grown root tips and had no caries or history of dental pulp treatment, in Weifang City People's Hospital, was chosen as the model. This study was conducted in accordance with the declaration of Helsinki and with approval from the Ethics Committee of Weifang People's Hospital. Written informed consent was obtained from the participant.

\section{Cone Beam Computed Tomography}

The volunteer underwent cone beam computed tomography (CBCT) under a peak tube voltage of $85 \mathrm{kV}$, tube current of $7 \mathrm{~mA}$, and radiation time of $6000 \mathrm{~ms}$. The data were stored in the Digital Imaging and Communications in Medicine (DICOM) format.

\section{Establishment of three-dimensional (3D) finite element model}

To establish and reconstruct the 3D models, the data were imported into Mimics software (version 18.0; Materialise, Leuven, Belgium); only the mandibular first molar was reserved.

\section{Experimental models}

With the basic model established for the mandibular first molar, experimental models with different degrees of tooth abrasion were produced as follows after deleting some of the data.

(1) Control group: 3D model of original mandibular first molar (Figure 1);

(2) Horizontal abrasion group: The molar was divided into four equal layers of the same vertical height from the mesio-occlusal plane to the contour line (Figure 2); 
(3) Tilt abrasion group: The molars were sectioned from the mesio-occlusal plane towards the distal gingival plane at the angles of $5^{\circ}, 10^{\circ}, 15^{\circ}$, and $20^{\circ}$ (Figure 3 ).

\section{Mechanical parameters of the material}

The elastic moduli and Poisson's ratios of the materials used in this study, shown in Table 1, were calculated according to [9].

Table 1

Mechanical parameters of various materials in the three-dimensional finite element model

\begin{tabular}{|lll|}
\hline Material & Elastic modulus (MPa) & Poisson's ratio \\
\hline Enamel & 83000 & 0.33 \\
\hline Dentin & 18600 & 0.31 \\
\hline Alveolar bone & 1370 & 0.3 \\
\hline Pulp & 2 & 0.45 \\
\hline Periodontal ligament & 6.89 & 0.45 \\
\hline
\end{tabular}

\section{Boundaries and loading}

The materials and tissues in the models were assumed to be continuous, homogeneous, and isotropic linear materials. Periapical tissue was completely fixed, which means that the movements in $X, Y, Z$ directions were all constrained.

After applying a vertical static load of $200 \mathrm{~N}$ to every model in MSC software, the stress distribution and instantaneous displacement were analyzed using finite element software (Mas.Marc).

\section{Results}

\section{Control group}

For the control group, the equivalent stresses and instantaneous displacements of dental tissues are shown in Figures 4 and 5. The maximum stress and maximum displacement values were $1.605 \mathrm{MPa}$ and $7.0198 \times 10^{-3} \mathrm{~mm}$, respectively. In the first molar, the stress was concentrated at the central fossa of the occlusal surface, and the greatest displacement contour was observed in the mesial crown, extending apically to the tooth neck.

\section{Horizontal abrasion group}

Equivalent stresses and instantaneous displacement distributions are shown in Figures 6 and 7. The maximum stress and maximum displacement values are shown in Table 2. In the permanent mandibular first molar, the stress was concentrated at the crown and the furcation area of the mesial and distal roots. 
With an increase in degree of abrasion, the greatest displacement contour gradually became wedgeshaped, with V-shaped contours passing through the tooth. The displacement contours became gradually biased to the mesio-occlusal surface of the tooth neck in model 1 and model 4 of the horizontal group.

Table 2

Maximum stress and maximum displacement of the four kinds of flat shear models for horizontal abrasion

\begin{tabular}{|lll|}
\hline Model & $\begin{array}{l}\text { Maximal stress value } \\
(\mathrm{MPa})\end{array}$ & Maximal displacement value $(\mathrm{mm})$ \\
\hline Model 1 & 5.45 & $6.498 \times 10^{-3}$ \\
\hline Model 2 & 5.799 & $5.010 \times 10^{-3}$ \\
\hline Model 3 & 5.3245 & $7.124 \times 10^{-3}$ \\
\hline Model 4 & 5.387 & $6.858 \times 10^{-3}$ \\
\hline
\end{tabular}

\section{Tilt abrasion}

Equivalent stress (Figure 8) and instantaneous displacement (Figure 9) in the tilt abrasion group show that the stress in this group was concentrated at the mesial root. When the tilt angle increased from $5^{\circ}$ to $15^{\circ}$, the stress concentration at the mesial root gradually increased. When the tilt angle reached $20^{\circ}$, the stress concentration at the mesial root decreased, instead being concentrated in the crown. The instantaneous displacement contour was positioned obliquely to the mesial and gingival side such that the cut surface forms an acute angle with the proximal root. The angle of the displacement contour line with the mesial root decreased with an increase in the tilt of abrasion. In the $15^{\circ}$ tilt-cut model, the displacement contours coincided with the long axis of the mesial root. In the $20^{\circ}$ tilt-cut model, the displacement contour was circular, and the maximum displacement was observed in the crown.

The maximum stress and maximum displacement values of the four models are shown in Table 3. The maximum stress value increased in the $15^{\circ}$ tilt model; however, this increase was significant in the $20^{\circ}$ tiltcut model. The maximum displacement value increased significantly in the $10^{\circ}$ and $15^{\circ}$ models, while in the $20^{\circ}$ model, the displacement was similar to that of the $5^{\circ}$ model. 
Table 3

Maximum stress and maximum displacement of the four bevel-tilted models for tilt abrasion

\begin{tabular}{|lll|}
\hline Model & $\begin{array}{l}\text { Maximal stress value } \\
(\mathrm{MPa})\end{array}$ & Maximal displacement value $(\mathrm{mm})$ \\
\hline Bevel $5^{\circ}$ & 5.125 & $6.491 \times 10^{-3}$ \\
\hline Bevel $10^{\circ}$ & 5.556 & $9.391 \times 10^{-3}$ \\
\hline Bevel $15^{\circ}$ & 6.339 & $11.41 \times 10^{-3}$ \\
\hline Bevel $20^{\circ}$ & 8.745 & $6.934 \times 10^{-3}$ \\
\hline
\end{tabular}

\section{Discussion}

To analyze the biomechanics of complex systems, such as a tooth, the application of complex research methods is required [13]. Finite element analysis has previously been used to successfully simulate clinically observed orthodontic tooth movement [14], as well as endodontic treatment protocols under static, dynamic, and multiple loading scenarios [15]. It is ideal for solving complex design problems. In this study, a finite element biomedical model was established for analyzing the mechanical stress distribution and transient displacement of the mandibular first molar upon application of a load on the occlusal surface using different abrasion models.

In this study, on the basis of the equivalent stress and instantaneous displacement in the control group, we found that stress from occlusal loading was concentrated in the central fossa, and that the maximal displacement contour was in the mesial crown near the cheek, extending down to the tooth neck. The stress on the first molar was concentrated at the crown and at the furcation of the mesial and distal roots. However, with the simulation of increased abrasion, the displacement contour gradually became Vshaped. This might account for a significant increase in the prevalence of wedge-shaped defects in patients with severe tooth wear [16].

In the wear models with tilted abrasion, the stress was concentrated in the mesial root, especially when the tilt angle was increased from $5^{\circ}$ to $15^{\circ}$. This concentration resulted from the vertical loading force, which would have been distributed differently with inclined wear of the tooth. This force can be decomposed into vertical and horizontal components of force, and with an increase in tilt angle, the magnitude of the horizontal component of force gradually increases, which in turn increases the load on the mesial root and makes it a stress concentration area. Thus, the occurrence of VRFs becomes more likely. This was also confirmed by the instantaneous displacement diagram, where the maximal contour was situated obliquely to the mesial and gingival side from the cut surface and formed an acute angle with the mesial root. With an increase of inclined wear, the angle between the displacement contour line and the mesial root decreases. In the $15^{\circ}$ inclined model, the displacement contours coincided with the 
long axis of the proximal root, showing that the mesial root was prone to vertical fractures. Thus, we propose that we could reduce the risk of vertical fractures caused by inclination via occlusal adjustment.

However, when the tilt angle was increased to $20^{\circ}$, the stress on the mesial root was reduced, and it was concentrated on the crown; additionally, the displacement contour became circular, and the maximum displacement appeared in the crown. Therefore, when the crown is worn out severely and the cusps become tilted, they become a stress concentration area that is prone to fracture. Appropriate jaw tuning could reduce the risk of such a cusp fracture.

According to previous work, there are several causes for vertical mesial root fracture of the first molar [17]. First, loading on a mandibular first molar that has some anatomical defects, like long vertical grooves on the distal side of the mesial root, whose oval canals bend to the distal side, would cause uneven stress on the tooth. A previous study [18] found that the diameter of a root that has vertically fractured was typically smaller than that of a normal root. Secondly, when the upper and lower first molars occlude, cusp inclination could disperse the loading force without causing severe damage to the tooth. However, when severe wear causes cusp disappearance, more force will be transferred to the root. Thirdly, when wear on the mandibular first molar crown is more severe on the distal side, a condition that might be related to the frequency, time, and intensity of loading, the likelihood of eruption of the distal side is higher than that on the mesial side due to compensation. Therefore, the crown is prone to tilt to the mesial side. Based on our models, when the distal tipping angle of the abrasion plane of the mandibular first molar increases, the angle between the instantaneous displacement contours and the tooth long axis becomes smaller. The mesial root suffers from the highest stress concentration, thereby increasing the possibility of longitudinal root fracture.

Our study does have some limitations to understanding the etiopathology of VRF. Various diets cause tooth abrasion in diverse ways [19], and we have modeled just two types of abrasion. In addition, the etiology of tooth wear is commonly multifactorial, and commonly a combination of erosion, attrition, abrasion, and abfraction [20]. Future modelling studies must be undertaken with a wider variety of tooth wear scenarios, as well as clinical studies aimed at preventing premature abrasion of the mandibular first molar.

In conclusion, this study model provided a theoretical basis for clinical grinding and jaw adjustment. In our models, the vertical fracture of the mesial root of the mandibular first molar was largely due to excessive tooth wear that results from occlusal tilt to the distal side and increased stress concentration on the mesial root. Therefore, we propose that VFRs could be prevented by adjusting the jaw.

\section{Declarations}

\section{ACKNOWLEDGEMENT}

This study was supported by Science and Technology Committee of Changning District, Shanghai (CNKW2020Y10). 


\section{DATA AVAILABILITY STATEMENT}

The raw data supporting the conclusions of this article will be made available by the authors, without undue reservation.

\section{ETHICS STATEMENTS}

The studies involving human participants were reviewed and approved by The Ethics Committee of Shanghai Jiaotong University. The patients/participants provided their written informed consent to participate in this study. No animal studies are presented in this manuscript. No potentially identifiable human images or data is presented in this study.

\section{AUTHOR CONTRIBUTIONS}

Conceived and designed the experiments: Haoming Zhao, Ke Guo; Performed the experiments: Guo Ke Wenjia Wei; Analyzed the data: Haoming Zhao, Shoufu Sun, Yueqi Shi, Huaqiang Zhao; Contributed reagents/materials/analysis tools: Ke Guo. All authors contributed to the article and approved the submitted version.

\section{References}

1. Rivera EM and Walton RE. Longitudinal tooth fractures: findings that contribute to complex endodontic diagnoses. Endod Topics 2007; 16: 82-111.

2. Taschieri S, Del Fabbro M, El Kabbaney A, Tsesis I, Rosen E and Corbella S. Microsurgical retreatment of an endodontically treated tooth with an apically located incomplete vertical root fracture: a clinical case report. Restor Dent Endod 2016; 41: 316-321.

3. Baageel TM, Allah EH, Bakalka GT, Jadu F, Yamany I, Jan AM, Bogari DF and Alhazzazi TY. Vertical root fracture: biological effects and accuracy of diagnostic imaging methods. J Int Soc Prev Community Dent 2016; 6 Suppl 2: S93-S104.

4. Seo DG, Yi YA, Shin SJ and Park JW. Analysis of factors associated with cracked teeth. J Endod 2012; 38: 288-292.

5. Johari M, Esmaeili F, Andalib A, Garjani S and Saberkari H. Detection of vertical root fractures in intact and endodontically treated premolar teeth by designing a probabilistic neural network: an ex vivo study. Dentomaxillofac Radiol 2016; 46(2):20160107.

6. Varshosaz M, Tavakoli MA, Mostafavi M and Baghban AA. Comparison of conventional radiography with cone beam computed tomography for detection of vertical root fractures: an in vitro study. $J$ Oral Sci 2010; 52: 593-597.

7. Yoshino K, Ito K, Kuroda M and Sugihara N. Prevalence of vertical root fracture as the reason for tooth extraction in dental clinics. Clin Oral Investig 2015; 19: 1405-1409.

8. Dai N, Hu J and Liu H. 3D simulation modeling of the tooth wear process. PLoS One 2015; 10 : e0134807. 
9. Wetselaar P, Wetselaar-Glas MJ, Koutris M, Visscher CM and Lobbezoo F. Assessment of the amount of tooth wear on dental casts and intra-oral photographs. J Oral Rehabil 2016; 43: 615-620.

10. Wetselaar $P$ and Lobbezoo F. The tooth wear evaluation system: a modular clinical guideline for the diagnosis and management planning of worn dentitions. J Oral Rehabil 2016; 43: 69-80.

11. Bardsley PF. The evolution of tooth wear indices. Clin Oral Investig 2008; 12 (Suppl 1): S15-S19.

12. Hove LH, Mulic A, Tveit AB, Stenhagen KR, Skaare AB and Espelid I. Registration of dental erosive wear on study models and intra-oral photographs. Eur Arch Paediatr Dent 2013; 14: 29-34.

13. Jakupović S, Anić I, Ajanović M, Korać S, Konjhodžić A, Džanković A and Vuković A. Biomechanics of cervical tooth region and noncarious cervical lesions of different morphology; three-dimensional finite element analysis. Eur J Dent 2016; 10: 413-418.

14. Kojima $\mathrm{Y}$ and Fukui $\mathrm{H}$. A finite element simulation of initial movement, orthodontic movement, and the centre of resistance of the maxillary teeth connected with an archwire. Eur J Orthod 2014; 36: 255-261.

15. Cinar D and Imirzalioglu P. The effect of three different crown heights and two different bone types on implants placed in the posterior maxilla: three-dimensional finite element analysis. Int $\mathrm{J}$ Oral Maxillofac Implants 2016; 31: e1-e10.

16. Dejak B, Mlotkowski A and Romanowicz M. Finite element analysis of stresses in molars during clenching and mastication. J Prosthet Dent 2003; 90: 591-597.

17. Abella F, Patel S, Durán-Sindreu F, Mercadé M and Roig M. Mandibular first molars with disto-lingual roots: review and clinical management. Int Endod J 2012; 45: 963-978.

18. Grieznis L, Apse P, Soboleva U. The effect of 2 different diameter cast post on tooth root fracture resistance in vitro. Stomatologija / issued by public institution "Odontologijos studija"2006;8(1):3032.

19. Karme A, Rannikko J, Kallonen A, Clauss M and Fortelius M. Mechanical modelling of tooth wear. J R Soc Interface 2016; 13: 20160399.

20. Kontaxopoulou I and Alam S. Risk assessment for tooth wear. Prim Dent J 2015; 4: 25-29.

\section{Figures}




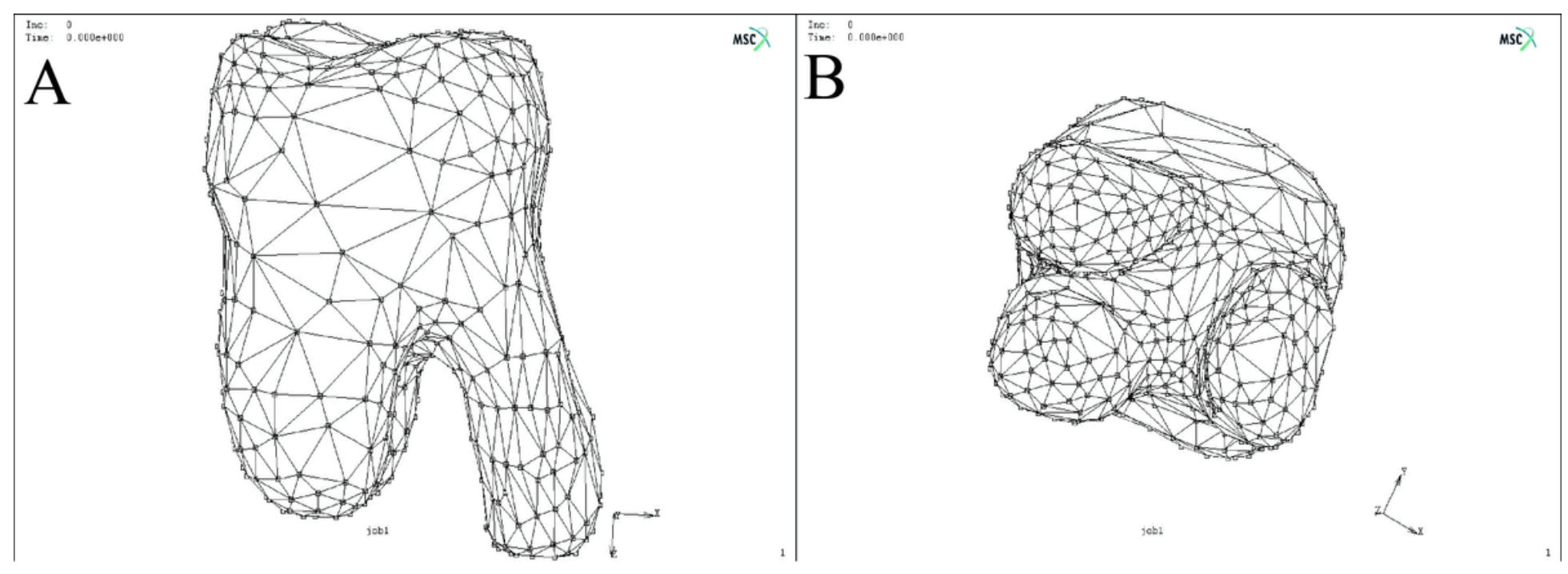

Figure 1

Models of the control group. A. Lateral view; B. Apical view.

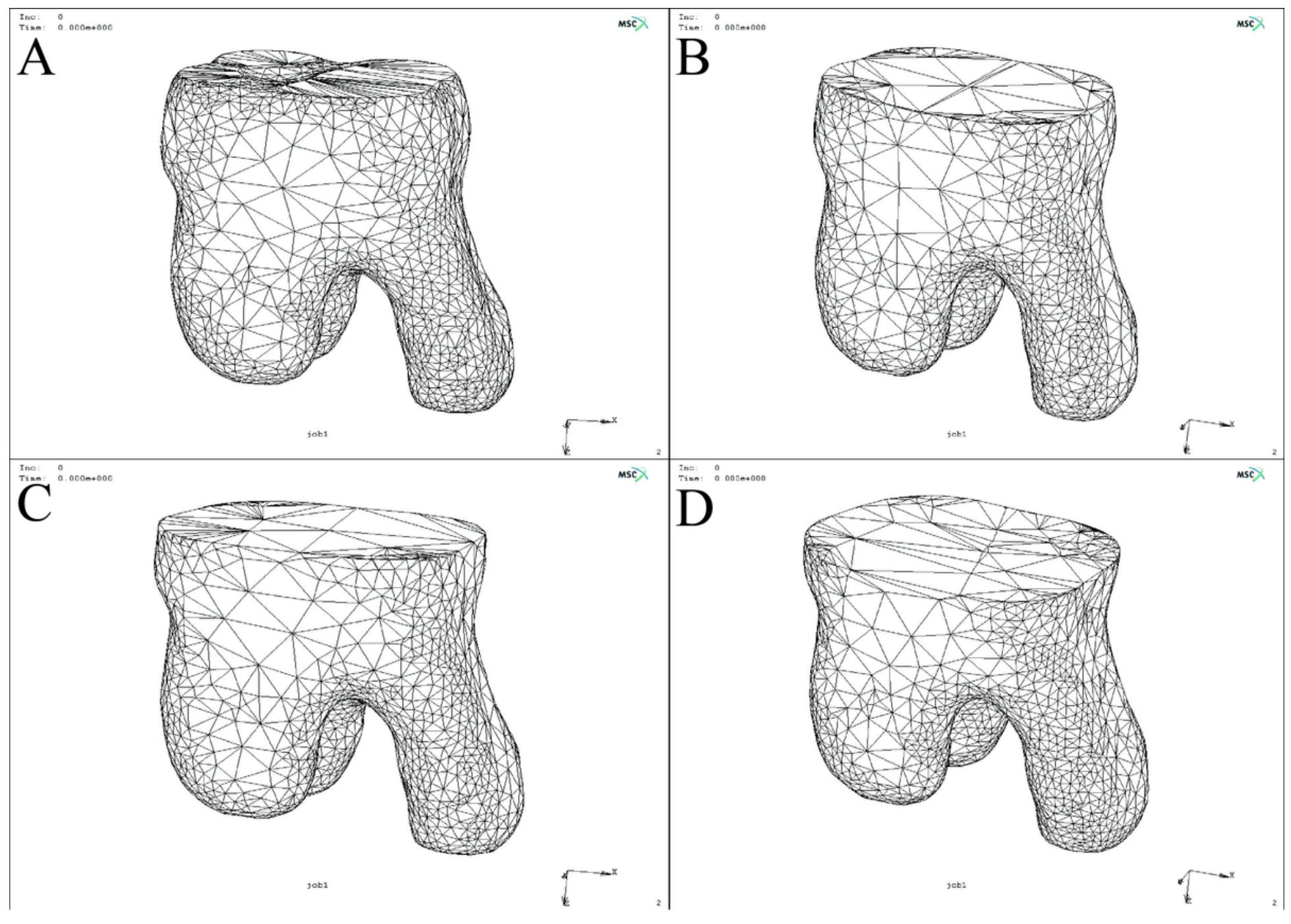

Figure 2 
Four models of horizontal abrasion, lateral views. A. Slice 1; B. Slice 2; C. Slice 3; D. Slice 4.

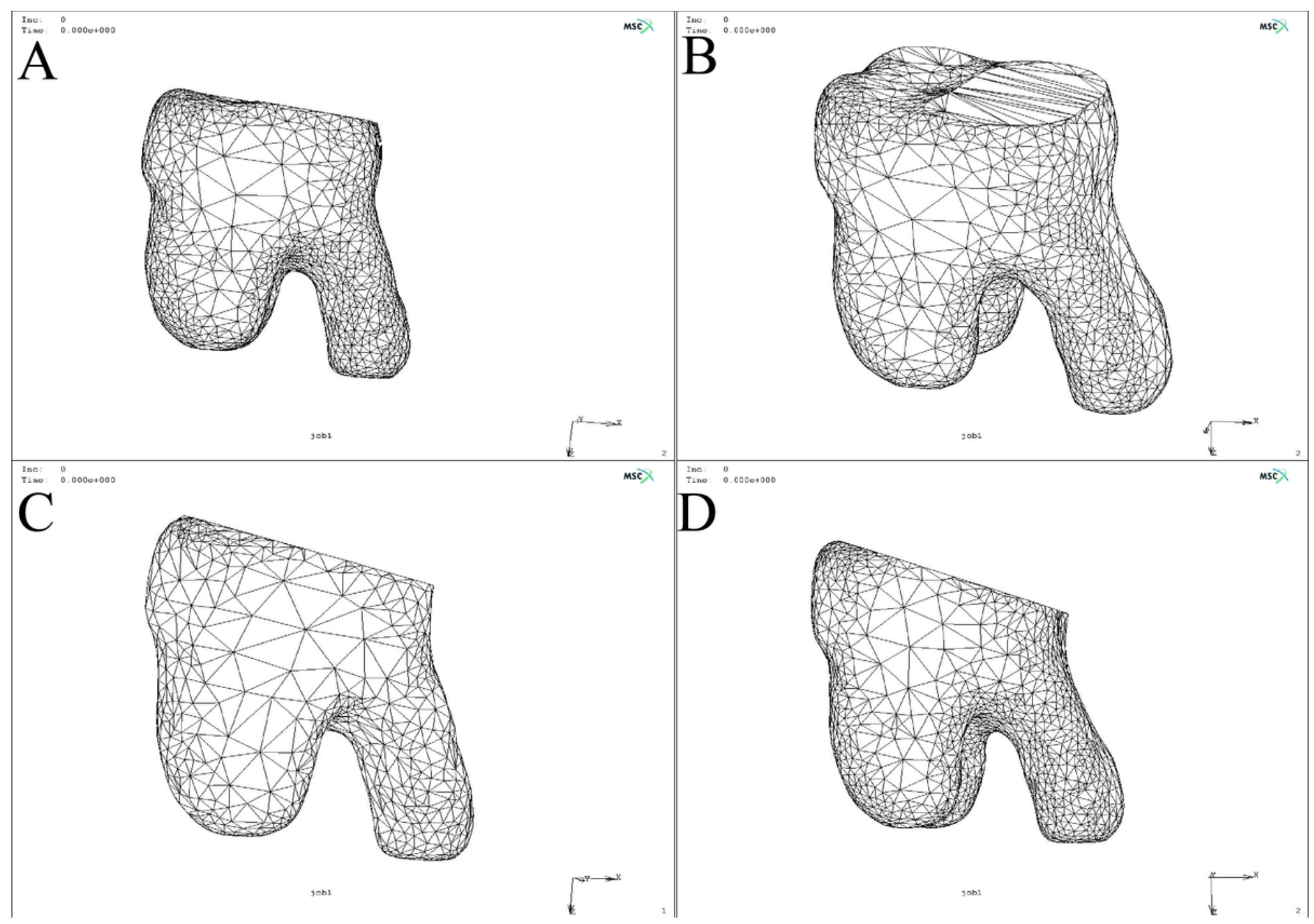

Figure 3

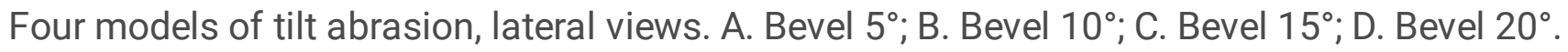




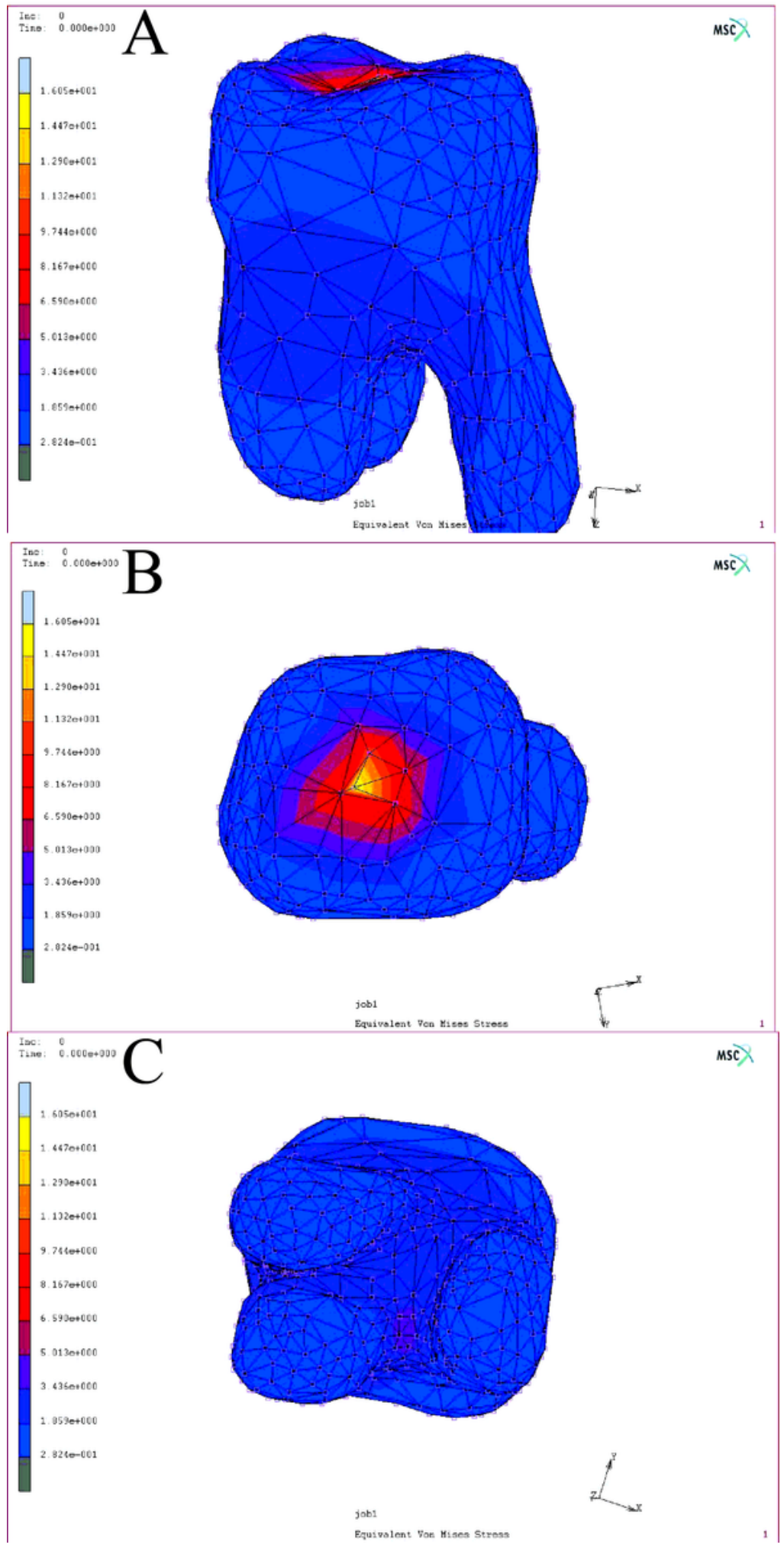

Figure 4

Equivalent stress in the control model. A. Lateral view; B. Occlusal view; C. Apical view. 


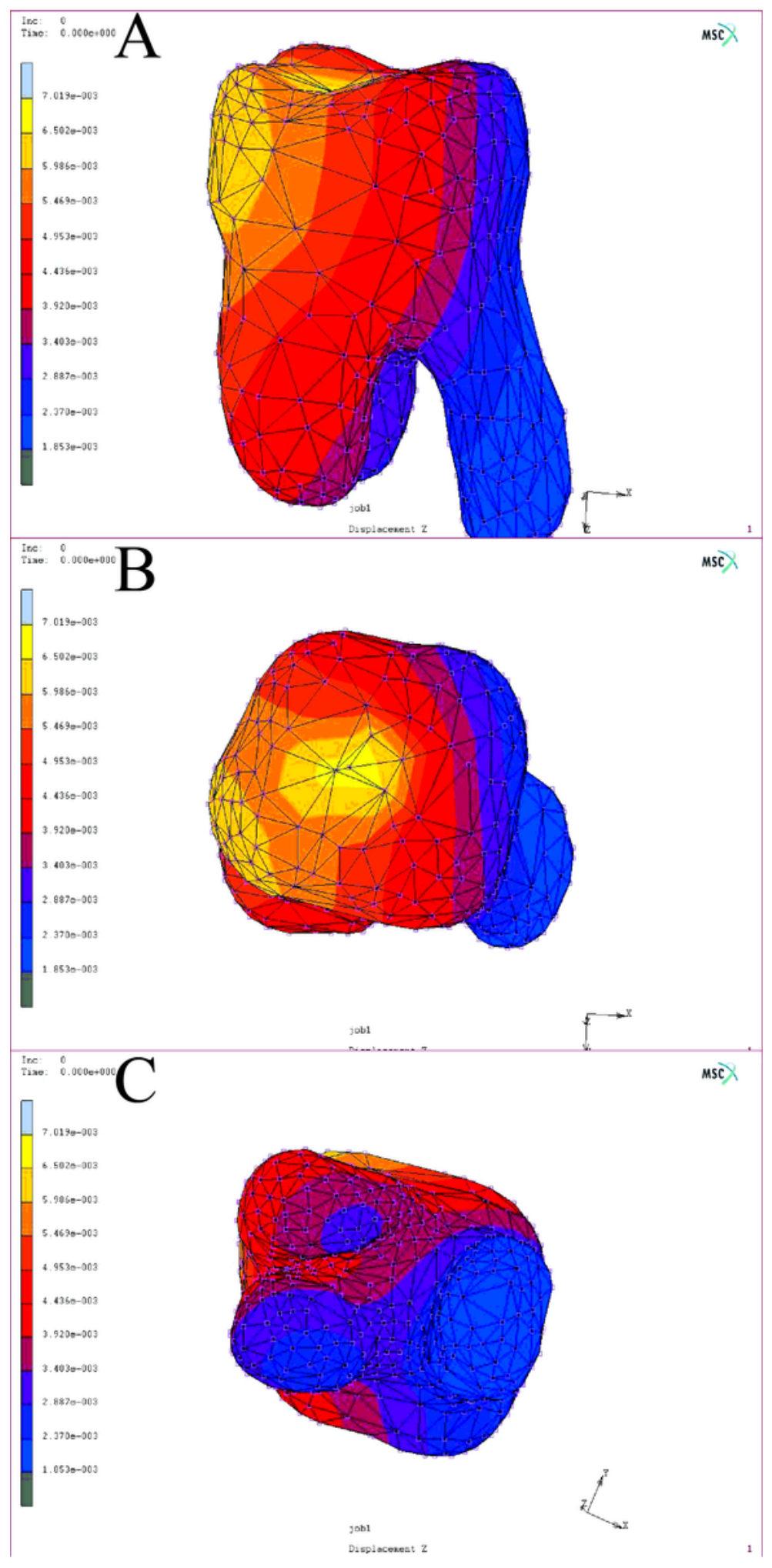

Figure 5

Instantaneous displacement in the control model. A. Lateral view; B. Occlusal view; C. Apical view. 


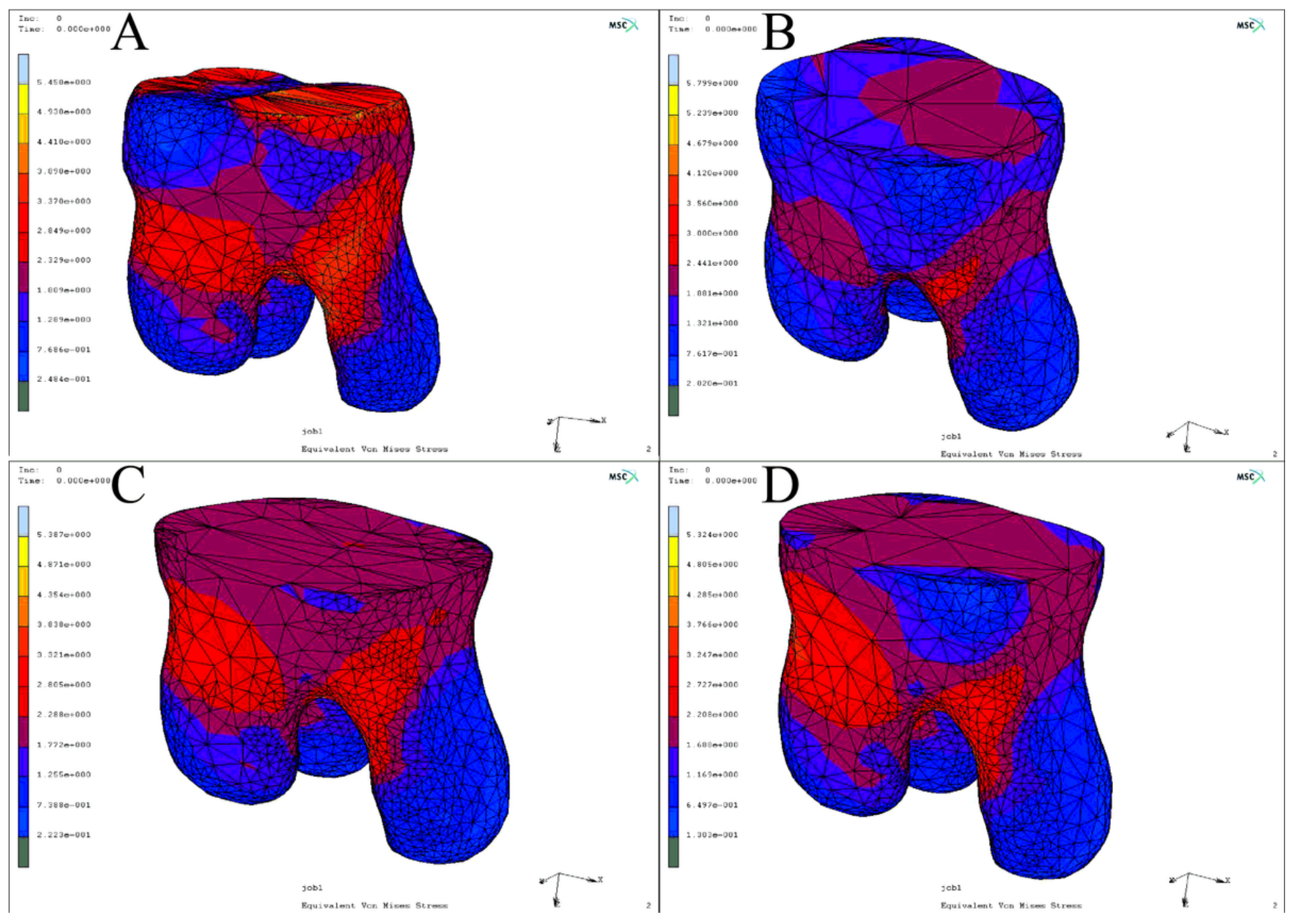

Figure 6

Equivalent stress in the four kinds of flat shear models in the horizontal abrasion group. A. Slice 1; B. Slice 2; C. Slice 3; D. Slice 4. 


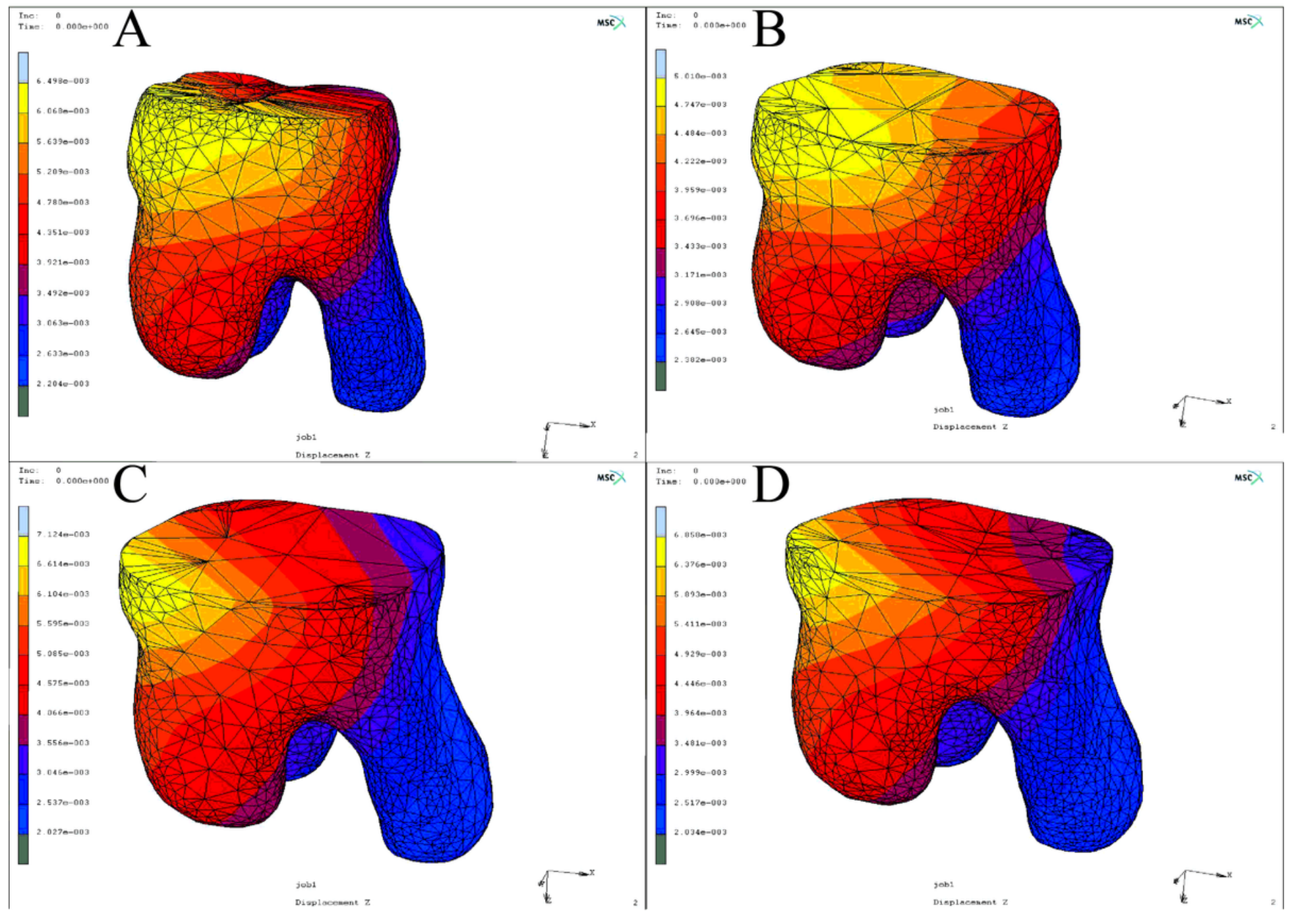

Figure 7

Instantaneous displacement in the four kinds of flat shear models in the horizontal abrasion group. A. Slice 1; B. Slice 2; C. Slice 3; D. Slice 4. 


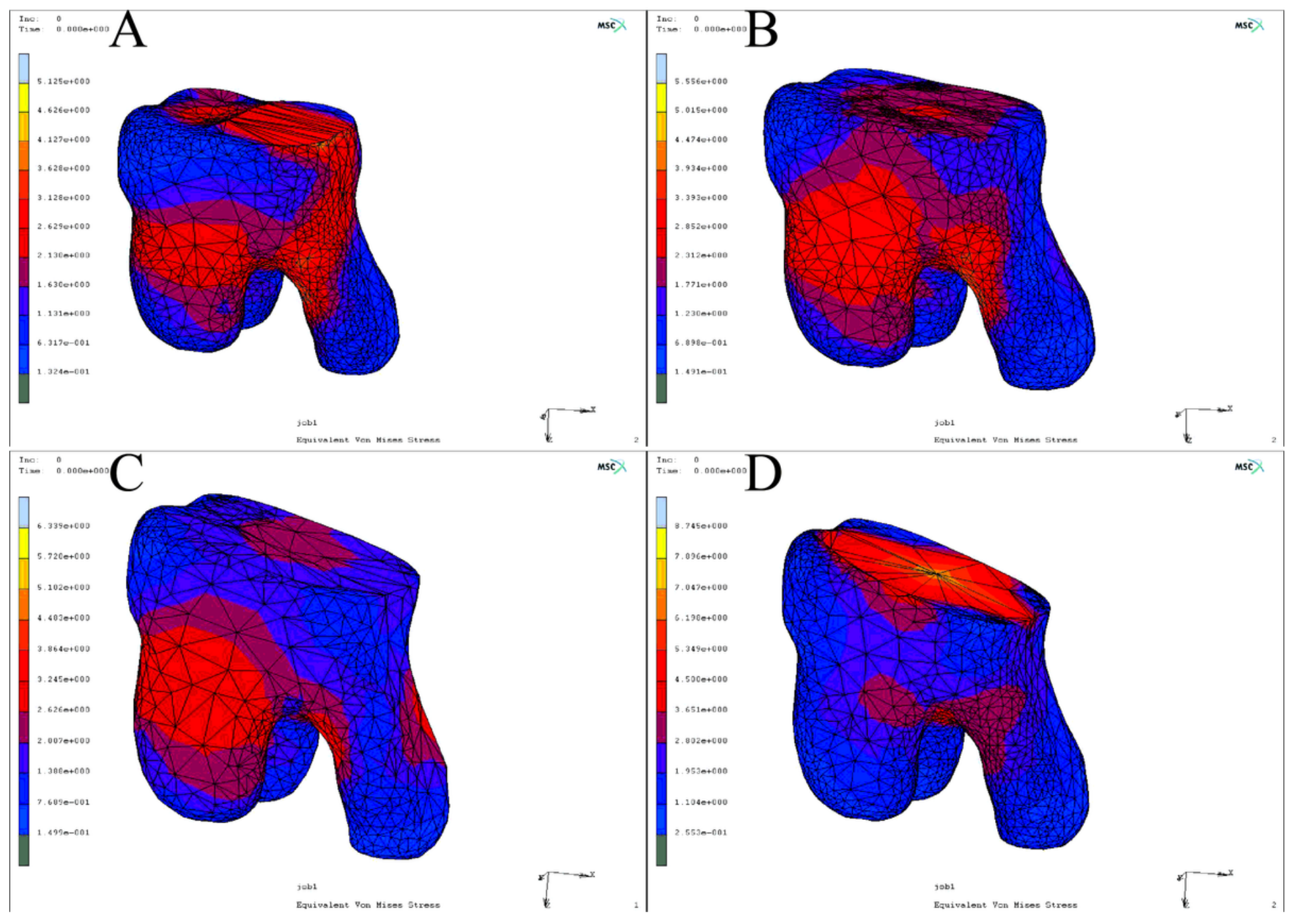

Figure 8

Equivalent stress models under tilt abrasion stress. A. Bevel 5\%; B. Bevel $10^{\circ} ;$ C. Bevel $15^{\circ}$; D. Bevel $20^{\circ}$. 


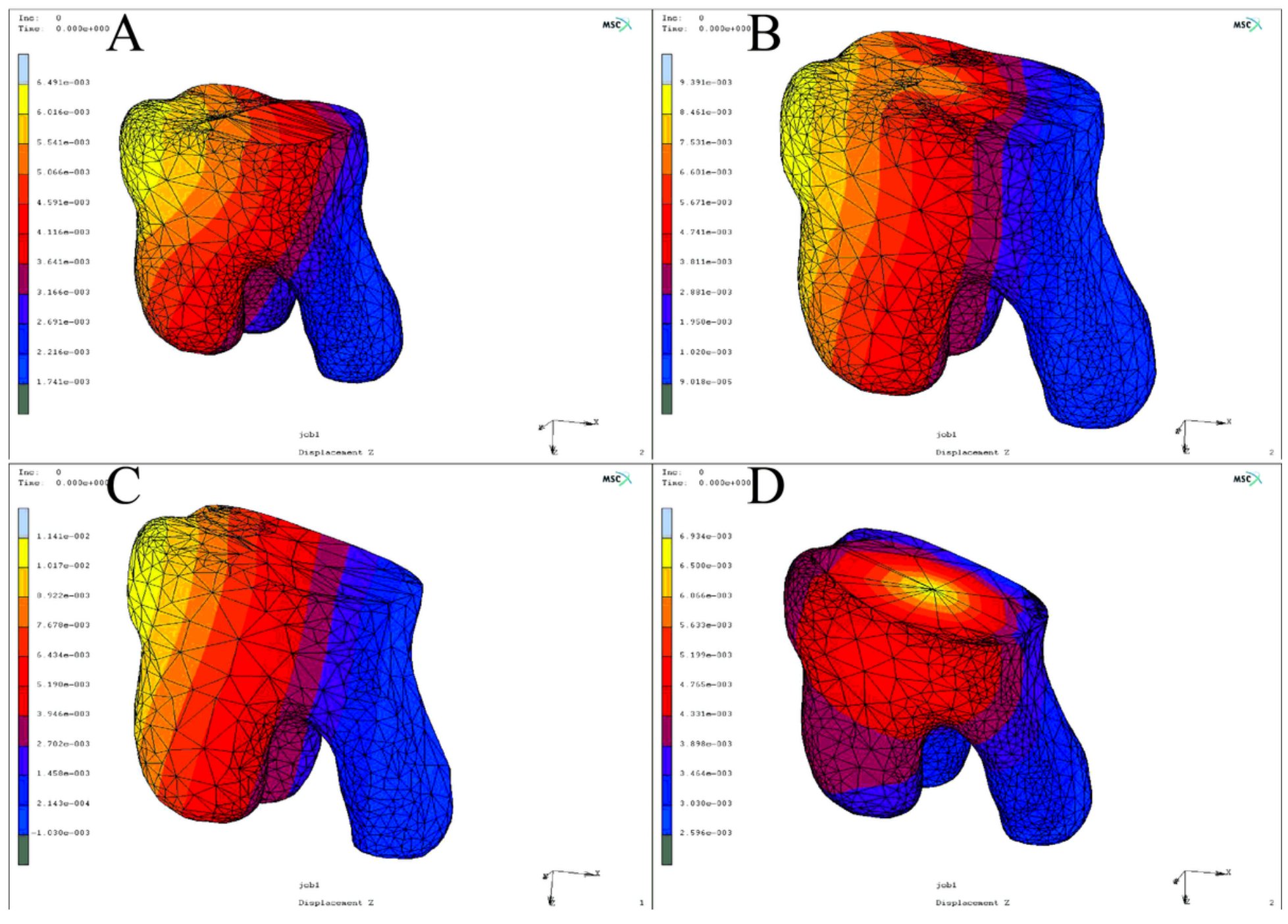

Figure 9

Instantaneous displacement in models in the tilt abrasion group. A. Bevel $5^{\circ}$; B. Bevel $10^{\circ} ; \mathrm{C}$. Bevel $15^{\circ}$; D. Bevel $20^{\circ}$. 